\title{
Effects of Different Types of Red Ginseng Marc and Fermented Red Koji Blend as Feed Additives on Blood Parameters and Egg Yolk Fatty Acid Profiles of Laying Hens
}

nAuthor(s)

\section{Lee $G D^{\prime}$}

Choi IH"

Department of Food Science and Biotechnology, Joongbu University, Geumsan-gun, Chungnam, 32713, South Korea.

" Department of Companion Animals and Animal Resources Science, Joongbu University, Geumsan-gun, Chungnam, 32713, South Korea.

\section{ABSTRACT}

The purpose of this study was to evaluate the effects of three different forms (powder, pellet, and coated pellet) of red ginseng marc (RGM) and fermented red koji (FRK) feed additive on the blood parameters and fatty acid (FA) profiles of laying hens, after two months of dietary supplementation. A number of 240 40-week-old Hy-line Brown laying hens were randomly designated to four dietary treatments, each with six replicates of 10 hens each, in a completely randomized design. The control group was fed a basal diet, and the other three treatments groups were fed $1 \%$ RGM powder mixed with FRK, pellets of $1 \%$ RGM with FRK, and coated pellets of $1 \%$ RGM with FRK. The powder and coated pellet diets contained the lowest total cholesterol and glucose levels, respectively, whereas the coated pellet diet contained the highest HDL cholesterol level, compared to the basal diet of the control group. For FA profiles, significant differences $(p<0.05)$ were observed among the groups with regard to the percentages of myristoleic acid, palmitoleic acid, margaric acid, margaroleic acid, stearic acid, oleic acid, $\alpha$-linolenic acid, eicosenoic acid, and docosahexaenoic acid. Moreover, addition of different forms of the RGM and FRK blend to the hen's diets showed an increase $(p<0.05)$ in the relative percentages of unsaturated fatty acids (UFA) and the UFA: SFA ratio and a decrease $(p<0.05)$ in the relative percentages of saturated fatty acids (SFA) in the egg yolk, compared to the control group. In conclusion, dietary coated pellets of RGM and FRK as a feed additive blend had a beneficial effect on serum cholesterol and FA profiles in laying hens.

\section{INTRODUCTION}

Red ginseng is widely used for the treatment of various diseases in Asian countries. It is reported to have many medicinal properties that are effective for the treatment of various health problems such as diabetes, insomnia, gastritis, dyspepsia, and the symptoms of overstrain, and fatigue. Red ginseng marc (RGM) is a byproduct of red ginseng extract and is used as an additive in poultry feed due to the positive effects of its components - especially saponins - on the growth, cholesterol levels, and meat quality of livestock (Ao et al., 2011; Kim et al., 2014). For example, studies have shown that broiler chickens fed diets supplemented with 3\% RGM had markedly decreased mortality and serum cholesterol levels, as well as improved meat quality, suggesting that RGM can be utilized in practical broiler diets (Kim et al., 2014).

Additionally, it has been reported that the ingestion of fermented red koji (FRK) (red koji rice fermented with the fungi Monascus) can have several therapeutic effects, such as improved digestion and lower serum cholesterol (Erdogrul \& Azirak, 2004). Fungi belonging to the genus Monascus, especially Monascus purpureus, produce a variety of pigments that, when ingested, can be beneficial to animals and 
humans. Red koji, processed by fermentation with M. purpureus, has long been consumed or exported in Asian countries as a dietary supplement and food additive because it contains monacolin $\mathrm{K}$, a bioactive compound (Erdogrul \& Azirak, 2004; Arunachalam \& Narmadhapriya, 2011). However, few studies have focused on the effects of different forms of a RGM and FRK blend as feed additives for laying hens. To the best of our knowledge, the present study is the first to develop a processing method to enhance the functionality of a RGM and FRK blend. Our previous research confirmed that the dietary addition of RGM and FRK had a positive influence on the growth performance, meat quality, and fatty acid (FA) profiles of broiler chickens (Chung \& Choi, 2016; Kim et al., 2016). Thus, in the present study, we supplemented the diet of laying hens with different forms of a RGM and FRK (powder, pellet and coated pellet) blend, rather than a single form, to evaluate its effects on the blood parameters and fatty acid composition of the egg yolk of laying hens. The aim of this study was to investigate the changes in the blood parameters and egg yolk FA profiles of laying hens fed diets with different forms of a RGM and FRK blend as feed additives.

\section{MATERIALS AND METHODS}

All procedures used in the current experiment were approved by the Animal Care and Use Committee of Heung Saeng Farm (Protocol No. 12-01, Euisung, South Korea).

\section{Preparation of red ginseng marc and fermented red koji samples}

The RGM and FRK used in this experiment were obtained from Yusim Co. (Yeongju, Korea). To process the materials, the wet RGM byproduct was air-dried at room temperature for $12 \mathrm{~h}$, followed by a hot air drying at $60^{\circ} \mathrm{C}$ for seven consecutive days until its moisture content reached $12 \%$. The samples were subsequently ground to prepare the fine RGM powder. The RGM and FRK powders were then thoroughly blended at a 9:1 ratio. Thereafter, pellets were prepared from the RGM and FRK blend using a pelleting mill (Kum Kang Eng., Daegu, South Korea). To prepare the coated pellets, the surfaces of the pellets were sprayed with canola oil using a small hand pump, and then allowed to dry for seven days. Finally, the three different forms of the RGM and FRK blend were placed in separate plastic bags in order to prevent cross contamination and were then refrigerated until use.

\section{Experimental design and birds}

In total, 240 Hy-line Brown laying hens, of 40 weeks age, were used in this experiment. A completely randomized experimental design was applied, consisting of four dietary treatment with six replicates (cages) with 10 hens each. The four dietary treatment groups were: the control group (basal diet), T1 (basal diet with 1\% RGM powder with FRK), T2 (basal diet with pellets of $1 \%$ RGM with FRK), and T3 (basal diet with coated pellets of $1 \%$ RGM with FRK). The hens were fed their designated dietary treatment for the entire eight-week experimental period.

The basal diet consistent of a standard commercial feed, containing 2,800 kcal metabolizable energy/ $\mathrm{kg}, 17.5 \%$ crude protein, and $3.8 \%$ calcium. The cages $(30-\mathrm{cm}$ wide $\times 80-\mathrm{cm}$ long $\times 50-\mathrm{cm}$ high) were equipped with nipple drinkers and trough feeders. Birds were housed in a windowless laying house at a temperature of approximately $21-23^{\circ} \mathrm{C}$ and a lighting regime of 16/8 $\mathrm{h}$ light/dark was applied. The housing facility was also equipped with a ventilation and temperature control system with variable settings to provide appropriate environmental conditions for the hens. Both feed and fresh water were offered ad libitum to all groups throughout the trial.

\section{Blood collection and cholesterol analysis}

At the end of the experimental period, 72 hens (18 hens per treatment group) were randomly selected after $6 \mathrm{~h}$ of feed deprivation. Blood was collected from the vena brachialis, located under the wing, using a sterilized syringe and needle. The samples were immediately transferred to vacuum tubes (Becton Dickinson Vacutainer Systems, Franklin Lakes, NJ, USA), and centrifuged at 2,000 $\times g$ for $30 \mathrm{~s}$ to separate the serum. Serum samples were stored at $-20^{\circ} \mathrm{C}$ (for up to two days) until used for determination of the serum cholesterol concentration. Serum total cholesterol (TC), high-density lipoprotein (HDL) cholesterol, and glucose levels were assayed by an enzymatic colorimetric method using an automatic analyzer (Hitachi 747, Hitachi Co., Tokyo, Japan) and direct enzymatic kits (Boehringer Mannheim, Germany).

\section{Fatty acid (FA) analysis}

At the end of the experiment, 72 eggs were randomly selected (18 eggs per treatment) to determine egg yolk fatty acid composition. To determine FA profiles, lipid was extracted from the egg yolk using a chloroform: methanol (2:1) solution as per the method of Folch et al. (1957). First, FAs were methylated with boron 
trifluoride after alkaline hydrolysis. The resulting fatty acid methyl esters (FAME) were analyzed by gas chromatography (GA-17A, Shimadzu, Tokyo, Japan) using a CP-Sil 88 column $(100 \mathrm{~m} \times 0.25 \mathrm{~mm} \times 0.2$ $\mu \mathrm{m})$ (Chrompack, Middelburg, the Netherlands). Gas chromatography was performed under the following conditions: the initial temperature was $180^{\circ} \mathrm{C}$, and was increased to $230^{\circ} \mathrm{C}$ at the rate of $1.5^{\circ} \mathrm{C} / \mathrm{min}$. The injector and detector temperatures were set at 240 and $260^{\circ} \mathrm{C}$, respectively. A $0.5-\mu \mathrm{L}$ sample was injected into the liner in split less mode, with a solvent delay of $5 \mathrm{~min}$. For the relative concentration, the peaks were identified by comparison of retention times with those of the corresponding standards acquired from Sigma (St. Louis, MO, USA). All peak areas measured in the FA were between C12:0 and C22:0. The FA levels were expressed as a percentage of the weight of all FAs detected.

\section{Statistical analysis}

All statistical analyses were performed using the generalized linear model (GLM) procedure of SAS Institute (2008) and analysis of variance (ANOVA). The same statistical methods used for laying hens as the experimental unit can also be used for pens (per 10 hens). The analysis of significant differences among the means was performed by Tukey's test, with the level of significance as $p<0.05$.

\section{RESULTS AND DISCUSSION}

\section{Blood parameters}

The results of blood parameters in laying hens fed diets with different forms of the RGM and FRK blend after two months is presented in Table 2. During the experimental period, significant differences $(p<0.05)$ were found in the TC, HDL cholesterol, and glucose levels among the four groups (Table 1). The control group had higher TC and glucose levels and lower HDL cholesterol levels than the groups fed the RGM and FRK blend (T1, T2, and T3). Regarding the groups fed the different forms of RGM and FRK, there were remarkable differences in serum cholesterol. The TC and glucose levels were lowest for the powder blend group (T1) and coated pellet group (T3), respectively, whereas the HDL cholesterol level was highest for the coated pellet group (T3). The LDL cholesterol level is not included in the results due to data error.

The results of this study suggest that dietary inclusion of RGM and FRK decreased serum TC and glucose levels in laying hens and increased serum HDL cholesterol level. This decrease in serum cholesterol levels is attributed to the bioactive components of red ginseng marc and fermented red koji, including saponin and monacolin $\mathrm{K}$. This is evidenced in a study by Oakenfull \& Sidhu (1989), in which saponins decreased blood cholesterol levels in birds; these functions were shown to be a consequence of the binding interactions between saponins and cholesterol in the intestinal bile. This suggests that cholesterol absorption is highly regulated and affected by particular compounds. Additionally, the monacolin $\mathrm{K}$ present in FRK acts as a potent inhibitor of HMGCOA reductase, which may also contribute to reduce serum cholesterol levels by lowering triglyceride (TG) levels (Wang et al., 1997). Another explanation is that fermentation is a useful tool for producing bioactive compounds in red koji. According to $\mathrm{Ng}$ et al. (2011), microbial fermentation can enrich Chinese herbs with vitamins, enzymes, and growth factors. Similarly, these results agree with the findings of Yan et al. (2011), who reported that dietary wild-ginseng adventitious root meal reduced the serum TC and TG levels. In addition, Jang et al. (2007) reported that dietary supplementation of fermented wild-ginseng culture byproduct decreased HDL cholesterol level in laying hens. Conversely, a study by Ao et al. (2011) shows that dietary supplementation with fermented red ginseng extract (FRGE) does not influence the TC of laying hens.

Table 1 - Blood parameters of laying hens fed diets with different types of red ginseng marc and fermented red kojifor 2 months.

\begin{tabular}{|c|c|c|c|c|c|c|}
\hline \multirow{2}{*}{ Parameters } & \multicolumn{4}{|c|}{ Treatment $^{1}$} & \multirow{2}{*}{$\mathrm{SEM}^{2}$} & \multirow{2}{*}{$p$-value } \\
\hline & Control & T1 & $\mathrm{T} 2$ & T3 & & \\
\hline Total cholesterol (mg/dL) & $131.50^{a}$ & $117.50^{b}$ & $126.42^{\mathrm{ab}}$ & $123.33^{\mathrm{ab}}$ & 2.927 & 0.0058 \\
\hline HDL-cholesterol (mg/dL) & $13.64^{b}$ & $15.92^{b}$ & $20.58^{a b}$ & $22.58^{a}$ & 2.058 & $p<0.001$ \\
\hline Glucose (mg/dL) & $238.75^{a}$ & $228.58^{\mathrm{ab}}$ & $219.08^{a b}$ & $214.58^{b}$ & 8.441 & 0.0429 \\
\hline
\end{tabular}

a-bMeans in the same row with no common superscript are significantly different $(p<0.05)$.

${ }^{1}$ Control: basal diet; T1: basal diet + 1\% \% red ginseng marc powder mixed with fermented red koji; T2: basal diet + pelleted 1\% red ginseng marc with fermented red koji; 73 : basal diet + coated pellets of $1 \%$ red ginseng marc with fermented red koji.

${ }^{2}$ Values are expressed as means \pm standard error. 
Table 2 - Fatty acid profiles of egg yolk of laying hens fed diets with different types of red ginseng marc and fermented red kojifor 2 months.

\begin{tabular}{|c|c|c|c|c|c|c|}
\hline \multirow{2}{*}{ Fatty acids (\%) } & \multicolumn{4}{|c|}{ Treatment $^{1}$} & \multirow{2}{*}{$\mathrm{SEM}^{2}$} & \multirow{2}{*}{$p$-value } \\
\hline & Con & T1 & $\mathrm{T} 2$ & T3 & & \\
\hline Myristic acid (C14:0) & 0.39 & 0.41 & 0.41 & 0.43 & 0.008 & 0.0567 \\
\hline Myristoleic acid (C14:1) & $0.10^{c}$ & $0.12^{b}$ & $0.12^{b}$ & $0.13^{\mathrm{a}}$ & 0.006 & 0.0014 \\
\hline Palmitic acid (C16:0) & 28.16 & 28.14 & 28.37 & 27.27 & 0.240 & 0.3658 \\
\hline Palmitoleic acid (C16:1) & $4.56^{c}$ & $4.85^{b}$ & $4.77^{\mathrm{bc}}$ & $5.14^{\mathrm{a}}$ & 0.120 & 0.0336 \\
\hline Margaric acid (C17:0) & $0.24^{c}$ & $0.25^{\text {bc }}$ & $0.26^{\mathrm{ab}}$ & $0.29^{a}$ & 0.011 & 0.0341 \\
\hline Margaroleic Acid (C17:1) & $0.28^{b}$ & $0.26^{c}$ & $0.27^{b c}$ & $0.33^{a}$ & 0.020 & 0.0006 \\
\hline Stearic acid (C18:0) & $15.59^{\mathrm{a}}$ & $11.95^{c}$ & $14.66^{b}$ & $11.03^{c}$ & 1.080 & $p<0.001$ \\
\hline Oleic acid (C18:1) & $31.08^{b}$ & $35.54^{\mathrm{a}}$ & $31.97^{b}$ & $35.85^{\mathrm{a}}$ & 2.220 & 0.0005 \\
\hline Linoleic acid (C18:2) & 15.20 & 14.11 & 14.88 & 14.91 & 0.231 & 0.5658 \\
\hline$\alpha$-linolenic acid (C18:3n-3) & $0.26^{c}$ & $0.33^{a}$ & $0.30^{\mathrm{b}}$ & $0.32^{\mathrm{ab}}$ & 0.020 & 0.0020 \\
\hline$\gamma$-linolenic acid (C18:3n-6) & 0.29 & 0.27 & 0.10 & 0.33 & 0.052 & 0.1073 \\
\hline Arachidic acid (C20:0) & 0.11 & 0.13 & 0.18 & 0.14 & 0.014 & 0.0693 \\
\hline Eicosenoic acid(C20:1) & $0.11^{\mathrm{b}}$ & $0.11^{\mathrm{b}}$ & $0.10^{\mathrm{b}}$ & $0.14^{a}$ & 0.009 & 0.0396 \\
\hline Eicosadienoic acid (C20:2) & 0.12 & 0.11 & 0.11 & 0.11 & 0.003 & 0.8051 \\
\hline Arachidonic acid (C20:4) & 2.59 & 2.54 & 2.58 & 2.60 & 0.011 & 0.8799 \\
\hline Eicosadienoic acid (C20:3n-6) & 0.05 & 0.06 & 0.06 & 0.06 & 0.003 & 0.2014 \\
\hline Eicosapentaenoic acid (C20:5n-3, EPA) & 0.01 & 0.01 & 0.01 & 0.01 & 0.000 & 0.2061 \\
\hline Docosapentaenoic acid (C22:5n-3, DPA) & 0.10 & 0.09 & 0.09 & 0.09 & 0.003 & 0.0700 \\
\hline Docosahexaenoic acid (C22:6n-3, DHA) & $0.76^{\mathrm{bc}}$ & $0.72^{c}$ & $0.77^{b}$ & $0.82^{\mathrm{a}}$ & 0.025 & 0.0416 \\
\hline Saturated fatty acid (SFA) & $44.49^{a}$ & $40.88^{b}$ & $43.88^{a}$ & $39.16^{c}$ & 2.154 & $p<0.001$ \\
\hline Unsaturated fatty acid (UFA) & $55.51^{c}$ & $59.12^{b}$ & $56.12^{c}$ & $60.84^{a}$ & 3.264 & $p<0.001$ \\
\hline UFA/SFA & $1.25^{c}$ & $1.45^{b}$ & $1.28^{c}$ & $1.56^{a}$ & 0.072 & $p<0.001$ \\
\hline
\end{tabular}

a-cMeans in the same row with no common superscript are significantly different $(p<0.05)$.

${ }^{1}$ Control: basal diet; T1: basal diet + 1\% \% red ginseng marc powder mixed with fermented red koji; T2: basal diet + pelleted 1\% red ginseng marc with fermented red koji; T3: basal diet + coated pellets of $1 \%$ red ginseng marc with fermented red koji.

${ }^{2}$ Values are expressed as means \pm standard error.

\section{Egg yolk fatty acid profiles}

Addition of different forms of RGM and FRK to the hens' diets resulted in a considerable variation in the relative FA compositions of the egg yolks (Table 2). Regarding the individual fatty acids, there were no significant differences $(p>0.05)$ in the percentages of myristic acid (C14:0), palmitic acid (C16:0), linoleic acid (C18:2), $\gamma$-linolenic acid (C18:3n-6), arachidic acid (C20:0), eicosadienoic acid (C20:2), arachidonic acid (C20:4), eicosadienoic acid (C20:3n-6), eicosapentaenoic acid (C20:5n-3, EPA), and docosapentaenoic acid (C22:5n-3, DPA) among all treatment groups. However, significant differences $(p<$ 0.05 ) were observed among the groups with regard to the percentages of myristoleic acid (C 14:1), palmitoleic acid (C16:1), margaric acid (C17:0), margaroleic acid (C17:1), stearic acid (C18:0), oleic acid (C18:1), $\alpha$-linolenic acid (C18:3n-3), eicosenoic acid (C20:1), and docosahexaenoic acid (C22:6n-3, DHA). Overall, the percentages of myristic acid, myristoleic acid, eicosadienoic acid, arachidonic acid, eicosadienoic acid, EPA, and DPA were similar for each treatment group, but there was a slight decrease or increase in the percentages of the other fatty acids for groups T1, T2, and T3, compared to the control group.

In the present study, addition of different forms of RGM and FRK to hens' diets resulted in an increase $(p<0.05)$ in the relative percentages of unsaturated fatty acid (UFA) and the UFA:SFA ratio, or a decrease $(p<0.05)$ in the relative percentages of saturated fatty acid (SFA) in the egg yolks, compared with the control group. These findings are consistent with those of previous studies, in which the ingestion of ginseng adventitious root meal reduced the percentage of SFAs and increased the percentage of UFAs, and the UFA:SFA ratio in the egg yolks of the treated hens (Yan et al., 2011). Additionally, observations of the egg yolks in this experiment revealed that the increase in the percentages of UFAs and the UFA:SFA ratio, and the decrease in the percentages of SFAs in all treatment groups after two months were in the following order: $\mathrm{T} 3>\mathrm{T} 1>\mathrm{T} 2>$ control group. This was primarily due to the significant increase in oleic acid (C18:1), which implies that the increase in the percentages of UFAs and decrease in the percentages of SFAs in the egg yolks as 
a result of dietary RGM and FRK supplementation may have an effect on the serum cholesterol concentration (Table 1). First, the other results can be explained by interactions between the bioactive components of RGM and FRK, as described by Oakenfull \& Sidhu (1989) and Wang et al. (1997). In a different study, El-Dakhakhny et al. (2000) reported that using black cumin seeds may have a favorable effect, either alone or in combination with other factors, on the synthesis of cholesterol in the liver, due to the presence of a high percentage of monounsaturated fatty acids. Second, the physical form of feed additives is considered to have a very significant impact on serum cholesterol levels and fatty acid profiles of egg yolks. In particular, coated and uncoated pellets can enhance bird performance by decreasing feed wastage and selective feeding, and increasing appetite and diet density (Jensen et al., 2010; Lv et al., 2015).

Regarding the health benefits of the treatment, it is known that both eicosapentaenoic acid (EPA) and docosahexaenoic acid (DHA) - n-3 long-chain polyunsaturated fatty acids (PUFA) - are the precursors of several metabolites that are potent lipid mediators, and are essential for the optimal functioning of cells, brains, tissues, and organs (Serhan et al., 2008). Moreover, the role of DHA is crucial in the early development stages of chicken, especially for eye and brain development (Lauritzen et al., 2001), and it is also known that EPA is a precursor of anti-inflammatory eicosanoids (Ajuyah et al., 2003). Thus, we suggest that feeding coated pellets of $1 \%$ RGM with FRK as feed additive is more effective in depositing DHA in the egg yolk and modifying the UFA composition of the egg yolk compared with the other forms of feed additives. Unfortunately, there are few studies focusing on the dietary effects of RGM and FRK on fatty acid profiles of poultry of other livestock.

\section{CONCLUSIONS}

It can be concluded that the supplementation of RGM and FRK to a hen's diet clearly resulted in decreased levels of serum cholesterol and SFAs, and increased PUFAs in the yolk. Thus, the use of RGM and FRK feed additives to lower the levels cholesterol and saturated fats in eggs may be more acceptable to consumers. In addition, an important advantage of RGM and FRK supplementation is that the technology required to prepare the powder, pellets, and coated pellets is easy and affordable, and therefore, feasible even for small farmers.

\section{ACKNOWLEDGEMENTS}

This research was financially supported by the Ministry of Trade, Industry and Energy (MOTIE) and Korea Institute for Advancement of Technology (KIAT, Grant number R0005536) through the Research and Development for Regional Industry.

\section{REFERENCES}

Ao X, Zhou TX, Kim HJ, Hong SM, Kim IH. Influence of fermented red ginseng extract on broiler and laying hens. Asian-Australian Journal of Animal Science 2011;24:993-1000.

Ajuyah AO, Cherian G, Wang $Y$, Sunwoo H, Sim JS. Maternal dietary FA modulate the long-chain n-3 PUFA status of chick cardiac tissue. Lipids 2003;38:1257-1261.

Arunachalam C, Narmadhapriya D. Monascus fermented rice and its beneficial aspects: a new review. Asian Journal of Pharmaceutical and Clinical Research 2011;4:29-31

Chung TH, Choi IH. Growth performance and fatty acid profiles of broilers given diets supplemented with fermented red ginseng marc powder combined with red koji .Brazilian Journal of Poultry Science 2016;18:733-738

El-Dakhakhny M, Mady NI, Halim MA. Nigella sativa oil protects against induced hepatotoxicity and improves serum lipid profile in rats. Arzneimittel Forschung - Drug Research 2000;50:832-836.

Erdogrul O, Azirak S. Review of the studies on the red yeast rice (Monascuspurpureus). Turkish Electronic Journal of Biotechnology 2004;2:37-49.

Folch J, Less M, Sloane-Stanley GH. A simple method for isolation and purification of total lipids from animal tissues. Journal of Biological Chemistry 1957;226:497-509.

Jang HD, Kim HJ, Cho JH, Chen YJ, Yoo JS, Min BJ, et al. Effect of dietary supplementation of fermented wild ginseng culture by products on egg productivity, egg quality, blood characteristics and ginsenoside concentration of yolk in laying hens. Korean Journal of Poultry Science 2007;34:271-278.

Jensen LS, Aumaitre A, Lee BD. Influence of pelleting on the nutritional needs of poultry. Asian-Australian Journal of Animal Science 2000;13:35-46

Kim SC, Lee GD, Choi IH. Breast meat quality of broilers fed fermented red ginseng marc powder mixed with red-koji during storage. Emirates Journal of Food and Agriculture 2016;28:283-287.

Kim YJ, Lee GD, Choi IH. Effects of dietary supplementation of red ginseng marc and $\alpha$-tocopherol on the growth performance and meat quality of broiler chicken. Journal of the Science of Food and Agriculture 2014;94:1816-1821.

Lauritzen L, Hansen HS, Jorgensen MH, Michaelsen KF. The essentiality of long chain $\mathrm{n}-3$ fatty acids in relation to development and function of the brain and retina. Progress in Lipid Research 2001;40:1-94.

Lv M, Yan L, Wang Z, An S, Wu M, Lv Z. Effects of feed form and feed particle size on growth performance, carcass characteristics and digestive tract development of broilers. Animal Nutrition 2015;1:252256 .

Ng CC, Wang CY, Wang YP, Tzeng WS, Shyu YT. Lactic acid bacteria fermentation on the production of functional antioxidant herbal Anoectochilus formosanus Hayata. Journal of Bioscience and Bioengineering 2011;111:289-293. 
Oakenfull D, Sidhu GS. Saponins. In: Cheeke PR, editor. Toxicants of plant origin. Boca Raton: CRC Press; 1989. p.97-141.

SAS Institute. SAS user's guide: statistics. Version 9.2. Cary: SAS Institute Inc.; 2008.

Serhan CN, Chiang N, Van Dyke TE. Resolving inflammation: dual antiinflammatory and pro-resolution lipid mediators. Nature Review Immunology 2008;8:349-61.
Wang J, Lu Z, Chi J, Wang W, Su M, Kou W. Multicenter clinical trial of the serum lipid-lowering effects of a Monascuspurpureus (red yeast) rice preparation from traditional Chinese medicine. Current Therapeutic Research 1997; 58:964-978.

Yan L, Meng QW, Ao X, Wang JP, Jang HD, Kim IH. Evaluation of dietary wild-ginseng adventitious root meal on egg production, egg quality, hematological profiles and egg yolk fatty acid composition in laying hens. Livestock Science 2011;140:201-205. 